\title{
The relationship of aerobic exercises and body weight reduction among regular physical fitness participants
}

\author{
Biruk Amare SORATE
}

Department of Sport Sciences, Jimma University, Jimma, Ethiopia.

Address correspondence to B. A. Sorate, amarebruk@gmail.com

\begin{abstract}
This study was aimed to examine the relationship of aerobic exercises and body weight reduction among regular physical fitness participants. The researcher used longitudinal experimental research design because participants were tested more than once test and trained for 12 weeks. Twenty subjects were purposively selected as a subject and randomly assigned in high intensity and moderate intensity group; whose age is in between twenty up to fifty years old and their body mass index (BMI) is $25 \mathrm{~kg} / \mathrm{m} 2$ and above. Both groups participated in high intensity and moderate intensity aerobics exercise in their respective groups three days per week for three consecutive months. Pre, during and post tests were taken accordingly to examine the improvements in each variables such as body weight, body mass index, waist circumference, hip circumference and waist hip ratio. Paired sample T-tests indicated a significant reduction of BWt and BMI in both groups of female and male participants at $\mathrm{p}$-value $(\mathrm{p}<0.01)$. Based on the results, it was concluded that both moderate intensity and high intensity aerobics exercise group had a positive effect on body weight reduction. It showed that aerobic exercise has a positive relationship on body weight reduction. Finally, the researcher recommends that regular aerobic exercise is a better means to reduce body weight.
\end{abstract}

Keywords: Aerobics exercise, body weight, physical fitness.

\section{INTRODUCTION}

Body composition assessment is an advanced method to investigate the status of the human body (3). It refers to the constituents of body-lean mass, fat mass and water, and is not only important for athletes, since an athlete's performance is partially influenced by the proportion of fat free mass (FFM) and fat mass (FM), but also for individuals of all ages, gender and ethnic groups (3).

Obesity is global and national epidemic worldwide $(1,21)$. It is a medical condition in which excess body fat has accumulated to the extent that it may have an adverse effect on health, leading to reduced life expectancy and/or increased health problems (8). Obesity is a leading but preventable cause of death worldwide, with increasing prevalence in adults and children, and it is one of the most serious public health problems of the twenty first century (2).

The problem of obesity and overweight requires remediation for prevention treating the incidence of chronic disease. Regular physical activity is one approach for obesity prevention and treatment (7), as participation in regular exercise reduces major health problems associated with excess adiposity $(5,9,20)$.

Most research evidence shows that physical exercise appears to play a critical role in body weight control management (11). They remarked that it's important to consider the duration, intensity and type of exercise that should be recommended for weight loss. However, aerobic exercise can be an important component of weight loss intervention, and therefore, commonly included as part of a comprehensive weight loss management program (19).

Physical activity can improve quality of life and is a critical component in reducing or eliminating health disparities through lowering resting heart rate and blood pressure, reducing hypertension, blood glucose, decreasing fat body mass and increasing lean body mass, bone mass and bone strength, increasing muscle strength, preventing arthritis, and reducing symptoms of mild or moderate depression $(10,15)$. 
Physical exercise has long been acknowledged as an important part of a healthy lifestyle, and recent scientific evidence has linked regular physical exercise to a wide range of physical health benefits. Research has demonstrated protective effects of varying strength between physical exercise and risk for several chronic diseases, including coronary heart disease, hypertension, non-insulin dependent diabetes mellitus, osteoporosis, and colon cancer (17).

In our country Ethiopia, because of a sedentary lifestyle using a fewer physical demands at work place or at home, makes imbalance between the amount of energy consumed (calories input) and the amount of energy expenditure (calories output) the body leads to store extra calories $(6,22)$. Similarly, most staff members of Jimma University are living a sedentary lifestyle are attacked by chronic disease, such as; coronary heart disease, hypertension, diabetes, over weight and obesity.

Thus, the researcher hypothesized that aerobic exercises could have a positive effect on body weight reduction. Therefore, the purpose of this study was to examine the relationship of aerobic exercises and body weight reduction among regular physical fitness participants.

\section{MATERIAL \& METHOD}

\section{Study Design and Area}

The researcher used longitudinal experimental research design because participants were tested more than once test and trained for 12 weeks. The study was conducted at the Jimma University Main Campus in fitness center. Jimma town located $350 \mathrm{~km}$ in southwest of Addis Ababa.

\section{Subjects}

The subjects in this study was 20 participants (10 males and 10 females) whose ages were in between 20 to 50 and their body mass index (BMI) is $25 \mathrm{~kg} / \mathrm{m} 2$ and above were purposively selected. They randomly grouped in to high intensity and moderate intensity group. The selected subjects were evaluated their baseline anthropometric measurements includes weight, height, body mass index (BMI), Waist circumference (WC) and Waist-hip ratio (WHR) before they were starting aerobic exercise training.

\section{Intervention (training) program}

The subjects who randomly assigned in high intensity and moderate intensity exercise groups were perform high and moderate intensity aerobic training in their respective groups for 12 weeks (moderate intensity approximately 60-65\% HR max and high intensity approximately $80-85 \%$ HR max) (23) that consisted of walking, jogging, running, and stretching; walking and running on treadmill, cycling, steps, aerobic dance, and others. During the 12 weeks, the program has three weekly training sessions, 60 minutes per session which includes 10 minutes of warm-up, 40 minutes of aerobic training and 10 minutes of cool down. The weekly training program was held on Monday, Thursday, and Friday (From 5:00 pm-6:00 pm). During the study period the feeding habits of the participants was as usual. The subjects were instructed to determine their training heart rates from $10 \mathrm{~s}$ pulse counts estimated several times during the exercise, and were advised to have a warm-up period of $15 \mathrm{~min}$ before and a $10 \mathrm{~min}$ slowdown period after the 30 min exercise period to avoid the risks of too vigorous changes of physical activity.

\section{Method of Data Collection}

For this study quantitative method of data collection was used. The data was collected through direct anthropometric measures from each of the subjects just before starting aerobic exercise, it was used as baseline. The second and the third tests were taken after the 6th and 12th weeks of aerobic exercise respectively. The tests were strictly measured and recorded by the researcher and classified with standard reference values.

Body weight measurement (BWt): The calibrated digital balanced beam a physician's scale in kilograms $(\mathrm{kg})$ to the nearest $0.1 \mathrm{~kg}$ will be used to measure the total body mass of an individual.

Body height measurement $(\mathrm{BHt})$ : The calibrated Stadiometer will be used to measure the total body height. The subjects will be measured without shoes.

Body mass index (BMI): This measurement will be taken purposely to calculate the percentage of total body height and weight ratio. It is an indirect measurement of body fat and can be calculated as our weight in kilogram divided by the square of our height in meters, $(\mathrm{kg} / \mathrm{m} 2)$. 
According to WHO classification, a BMI of 30 $\mathrm{kg} / \mathrm{m}^{2}$ or more indicates obesity, while the cut-off point for overweight is $25 \mathrm{~kg} / \mathrm{m}^{2}$. This is calculated as: weight in $(\mathrm{kg})$ divided by height in $\left(\mathrm{m}^{2}\right)=$ body mass index (BMI).

Waist circumference (WC): This technique is used to measure the fat accumulation around abdomen using a Gulick tape meter two times. While the measurements are takes place subjects standing with light garments and waist is measured at the level midway between the lower rib margin and the iliac crest, with the participant breathing out gently.

Waist-hip ratio (WHR): This measurement is also done by using a Gulick tape meter two times, and it is probably the most used method for assessing abdominal fat distribution so far. The recommended procedure for the measurement of the WHR is following: Subject standing with light garments waist is measured at the level midway between the lower rib margin and the iliac crest, with the participant breathing out gently. Hip is measured as the maximum circumference over the buttocks.

\section{Ethical Issues and Code of Conduct}

The research was conducted as per rules, policies, and research ethics of Jimma University. Permission was obtained from the office of research and postgraduate coordinator. The purpose of the study was explained to the subjects to obtain verbal consent and written consent form was read and signed by each subjects. Then an informed and written consent was received from each study subjects. The privacy of the participants was guaranteed in risk of any harm.

\section{Data Analysis}

Descriptive statistics were produced for each of the parameters. The results were presented as mean \pm SD. Paired sample T-tests were used to find out the significant difference in anthropometric tests. The significance level was set at $\mathrm{P}<0.05$ for each of the statistical tests performed. The SPSS 20 software was used for the statistical analysis.

\section{RESULTS}

\section{Characteristics of the Subjects}

The subjects of the study were both academic and administrative staffs of Jimma University, who are working at main campus, from different background, heterogeneous in their activities. 100\% of the selected subjects grouped in high intensity group and moderate intensity group were perform high and moderate intensity aerobic training in their respective groups for 12 weeks. There was no additional performance assessing treatment provided for those groups during the study. Both groups were ate the food as usual throughout the study period.

Table 2 showed that a significant changes in the reduction of BWt and BMI in both groups of female and male participants. BWt of female participants reduces from 74 to $68.6 \mathrm{~kg}$ in HIG and 74 to $70.4 \mathrm{~kg}$ in MIG. In the other hand, BW of male participants reduces from 81.8 to $76.8 \mathrm{~kg}$ in HIG and 84.8 to $81.2 \mathrm{~kg}$ in MIG. BMI of female participants reduces from 27 to $25.1 \mathrm{~kg} / \mathrm{m} 2$ in HIG and 28 to $26.9 \mathrm{~kg} / \mathrm{m} 2$ in MIG. However, BMI of male participants reduces from 27.8 to $26.1 \mathrm{~kg} / \mathrm{m} 2$ in HIG and 27 to $25.9 \mathrm{~kg} / \mathrm{m} 2$ in MIG.

Table 1. Baseline characteristics of the subjects treated with 12 weeks aerobic exercise in high intensity group (HIG) and moderate intensity group (MIG).

\begin{tabular}{lcccc}
\hline \multirow{2}{*}{ Parameters } & \multicolumn{2}{c}{ HIG } & MIG \\
\cline { 2 - 5 } & Female & Male & Female & Male \\
Age & $31.40 \pm 3.16$ & $31.40 \pm 3.16$ & $33 \pm 4.13$ & $33 \pm 4.13$ \\
BWt $(\mathrm{Kg})$ & $74 \pm 9.03$ & $81.8 \pm 3.77$ & $74 \pm 8.6$ & $84.8 \pm 2.39$ \\
BMI $(\mathrm{Kg} / \mathrm{m} 2)$ & $27 \pm 1.99$ & $27.8 \pm 1.52$ & $28 \pm 1.51$ & $27 \pm 1.45$ \\
WC $(\mathrm{Cm})$ & $91.6 \pm 7.6$ & $103.6 \pm 2.51$ & $94 \pm 7.91$ & $105 \pm 3.61$ \\
HC $(\mathrm{Cm})$ & $106.6 \pm 8.35$ & $101.6 \pm 5.59$ & $0.89 \pm 0.08$ & $102.8 \pm 3.11$ \\
WHR $(\%)$ & $0.86 \pm 0.06$ & $1.02 \pm 0.05$ & & $1.02 \pm 0.06$ \\
\end{tabular}


Table 2. Descriptive statistics and T-test result of BWt and BMI of HIG and MIG.

\begin{tabular}{|c|c|c|c|c|c|c|c|c|c|c|c|}
\hline \multirow{2}{*}{ Variables } & \multicolumn{6}{|c|}{ Female } & \multicolumn{5}{|c|}{ Male } \\
\hline & Tests & $\mathrm{df}$ & Mean \pm SD & Mean Diff. & $\mathrm{t}$ & $\mathrm{p}$ & $\mathrm{df}$ & Mean \pm SD & Mean Diff. & $\mathrm{t}$ & $\mathrm{p}$ \\
\hline \multicolumn{12}{|c|}{ High Intensity Group (HIG) } \\
\hline BWt & PT & 4 & $74 \pm 9.03$ & \multirow{2}{*}{$5.40^{*}$} & \multirow{2}{*}{10.59} & \multirow{2}{*}{0.001} & 4 & $81.8 \pm 3.77$ & \multirow{2}{*}{$5.00^{*}$} & \multirow{2}{*}{11.18} & \multirow{2}{*}{0.000} \\
\hline$(\mathrm{Kg})$ & PoT & 4 & $68.6 \pm 7.99$ & & & & 4 & $76.8 \pm 3.27$ & & & \\
\hline \multirow{2}{*}{ BMI (Kg/m2) } & PT & 4 & $27 \pm 1.99$ & \multirow{2}{*}{$1.96^{*}$} & \multirow{2}{*}{13.15} & \multirow{2}{*}{0.000} & 4 & $27.8 \pm 1.52$ & \multirow{2}{*}{$1.70^{*}$} & \multirow{2}{*}{10.61} & \multirow{2}{*}{0.000} \\
\hline & PoT & 4 & $25.1 \pm 1.69$ & & & & 4 & $26.1 \pm 1.29$ & & & \\
\hline \multicolumn{12}{|c|}{ Moderate Intensity Group (MIG) } \\
\hline BWt & PT & 4 & $74 \pm 8.6$ & \multirow{2}{*}{$3.60^{*}$} & \multirow{2}{*}{9.00} & \multirow{2}{*}{0.001} & 4 & $84.8 \pm 2.39$ & \multirow{2}{*}{$0.69^{*}$} & \multirow{2}{*}{13.92} & \multirow{2}{*}{0.000} \\
\hline$(\mathrm{Kg})$ & PoT & 4 & $70.4 \pm 8.17$ & & & & 4 & $81.2 \pm 2.28$ & & & \\
\hline BMI & PT & 4 & $28 \pm 1.51$ & \multirow{2}{*}{$1.37^{*}$} & \multirow{2}{*}{11.46} & \multirow{2}{*}{0.000} & 4 & $27 \pm 1.45$ & \multirow{2}{*}{$1.13^{*}$} & \multirow{2}{*}{11.84} & \multirow{2}{*}{0.000} \\
\hline$(\mathrm{Kg} / \mathrm{m} 2)$ & PoT & 4 & $26.9 \pm 1.54$ & & & & 4 & $25.9 \pm 1.64$ & & & \\
\hline
\end{tabular}

${ }^{*}$ Mean \pm SD in the same columns in each parameter are significantly different $(\mathrm{p}<0.05)$.

Table 3. Descriptive statistics and T-test result of WC and HC of HIG and MIG.

\begin{tabular}{|c|c|c|c|c|c|c|c|c|c|c|c|}
\hline \multirow{2}{*}{ Variables } & \multicolumn{6}{|c|}{ Female } & \multicolumn{5}{|c|}{ Male } \\
\hline & Tests & $\mathrm{df}$ & Mean \pm SD & Mean Diff. & $\mathrm{t}$ & $p$ & $\mathrm{df}$ & Mean \pm SD & Mean Diff. & $\mathrm{t}$ & $p$ \\
\hline \multicolumn{12}{|c|}{ High Intensity Group (HIG) } \\
\hline \multirow{2}{*}{$\mathrm{WC}(\mathrm{cm})$} & PT & 4 & $91.6 \pm 7.6$ & \multirow{2}{*}{$7.60^{*}$} & \multirow{2}{*}{14.90} & \multirow{2}{*}{0.000} & 4 & $103.6 \pm 2.51$ & \multirow{2}{*}{$8.20^{*}$} & \multirow{2}{*}{12.36} & \multirow{2}{*}{0.000} \\
\hline & PoT & 4 & $84 \pm 6.89$ & & & & 4 & $95.4 \pm 2.07$ & & & \\
\hline \multirow{2}{*}{$\mathrm{HC}(\mathrm{cm})$} & PT & 4 & $106.6 \pm 8.35$ & \multirow{2}{*}{$4.00^{*}$} & \multirow{2}{*}{8.94} & \multirow{2}{*}{0.001} & 4 & $101.6 \pm 5.59$ & \multirow{2}{*}{$4.00^{*}$} & \multirow{2}{*}{12.64} & \multirow{2}{*}{0.000} \\
\hline & PoT & 4 & $102.6 \pm 8.02$ & & & & 4 & $97.6 \pm 5.46$ & & & \\
\hline \multirow{2}{*}{ WHR } & PT & 4 & $0.86 \pm 0.06$ & \multirow{2}{*}{$0.04^{*}$} & \multirow{2}{*}{17.96} & \multirow{2}{*}{0.000} & 4 & $1.02 \pm 0.05$ & \multirow{2}{*}{$0.04^{*}$} & \multirow{2}{*}{8.57} & \multirow{2}{*}{0.000} \\
\hline & PoT & 4 & $0.82 \pm 0.06$ & & & & 4 & $0.98 \pm 0.05$ & & & \\
\hline \multicolumn{12}{|c|}{ Moderate Intensity Group (MIG) } \\
\hline \multirow{2}{*}{$\mathrm{WC}(\mathrm{cm})$} & PT & 4 & $94 \pm 7.91$ & \multirow{2}{*}{$7.20^{*}$} & \multirow{2}{*}{12.34} & \multirow{2}{*}{0.000} & 4 & $105 \pm 3.61$ & \multirow{2}{*}{$7.20^{*}$} & \multirow{2}{*}{19.24} & \multirow{2}{*}{0.000} \\
\hline & PoT & 4 & $86.8 \pm 7.50$ & & & & 4 & $97.8 \pm 3.63$ & & & \\
\hline \multirow{2}{*}{$\mathrm{HC}(\mathrm{cm})$} & PT & 4 & $107.2 \pm 9.58$ & \multirow{2}{*}{$3.60^{*}$} & 900 & 0.001 & 4 & $102.8 \pm 3.11$ & $380^{*}$ & 1900 & 0,000 \\
\hline & PoT & 4 & $103.6 \pm 8.82$ & & 9.00 & 0.001 & 4 & $99 \pm 2.92$ & $3.80^{n}$ & & \\
\hline WHR & PT & 4 & $0.89 \pm 0.08$ & $0.04^{*}$ & 17.96 & 0.000 & 4 & $1.02 \pm 0.06$ & $0.03^{*}$ & 13.88 & 0.000 \\
\hline VITK & PoT & 4 & $0.84 \pm 0.08$ & $0.04^{\circ}$ & 17.96 & 0.000 & 4 & $0.99 \pm 0.06$ & & & \\
\hline
\end{tabular}

${ }^{*} \mathrm{P}<0.05$

Mean \pm SD in the same columns in each parameter with different* superscripts are significantly different ( $<<0.05)$, Total cholesterol (TC), Triglyceride (TG), High density lipoprotein (HDL) and Low density lipoprotein (LDL)

Table 3 showed that a significant changes in the reduction of WC and $\mathrm{HC}$ in both groups of female and male participants. WC of female participants reduces from 91.6 to $84 \mathrm{~cm}$ in HIG and 94 to $86.8 \mathrm{~cm}$ in MIG. In the other hand, WC of male participants reduces from 103.6 to $95.4 \mathrm{~cm}$ in HIG and 105 to $97.8 \mathrm{~cm}$ in MIG. HC of female participants reduces from 106.6 to $102.6 \mathrm{~cm}$ in HIG and 107.2 to $103.6 \mathrm{~cm}$ in MIG. However, HC of male participants reduces from 101.6 to $97.6 \mathrm{~cm}$ in HIG and 102.8 to $99 \mathrm{~cm}$ in MIG.

Table 3 also showed that a significant changes in the reduction of WHR in both groups of female and male participants. WHR of female participants reduces from 0.86 to 0.82 in HIG and 0.89 to 0.84 in MIG. In the other hand, WHR of male participants reduces from
1.02 to 0.98 in HIG and 1.02 to 0.99 in MIG.

\section{DISCUSSIONS}

This study was aimed to report on the basis of longitudinal study, however, we have to keep in mind that conclusions have been drawn. Thus, this study was tried to examine the relationship of aerobic exercises and body weight reduction among regular physical fitness participants of Jimma University fitness center.

The data in table 2 showed that there were significant changes in the reduction of body weight and BMI in both groups of female participants. Table 2 also indicated that the male participants' body weight and BMI were highly reduced. This was due to the 
training program they were engaged in the gymnasium. Similarly, recent study clearly shows that exercise increases the total energy needs, thus forcing the body to remove fat from the fat deposit to supply the additional energy and causing loss of weight (13).

However, a better change was recorded under high intensity exercise group than moderate intensity exercise group. This was because of more calories were burned during high intensity aerobics physical exercise compared to moderate intensity aerobics physical exercise. This result was in agreement with the findings (14) reported that high intensity in exercise regimen had impact on the body composition (lean body weight, \% body fat and body density) of the participants as compared to moderate intensity exercise (14). Another finding also noted that aerobics exercise can be an important component of weight loss intervention and therefore, commonly included as part of a comprehensive weight loss management program (12).

The data in table 3 revealed that the results of waist and hip circumference in both groups of female participants were progressively changed from the pretest to post test of the experimental period. The better improvement was also recorded in both groups of male participants. Even though the improvement had been observed in both groups, the high intensity groups showed a great change as compared to moderate intensity group participants. This result was also directly similar to the previous finding (18) reported that high intensity intermittent exercise training induced greater visceral fat loss compared to moderate intensity exercise (18). Another finding by (4) also reported that the high intensity intermittent exercise has greater fat loss than moderate intensity intermittent exercise (4).

As table 3 clearly showed that there were significant changes in waist hip ratio from pre to post training tests in both groups of female participants. Also as indicated in table 3 the results of waist hip ratio, both groups of male participants were showed significant change at post tests. However, the result obtained from both groups the high intensity aerobics exercise group was showed a better mean change than the moderate aerobics group. Depending on this result it is possible to conclude that high intensity aerobics exercise has a positive effect on the reduction of fat around the waist, abdomen and trunk areas of the body. This result is directly similar with the finding of
$(16,4)$; they noted that high intensity intermittent exercise performed three times per week for 12 weeks resulted in a significant reduction in total body, abdominal, trunk and visceral fat and a significant increase in fat free mass of overweight person $(4,16)$.

Based on the major findings of the study the following points are stated as conclusion. All variables clearly showed that the better mean changes were recorded in post training. Regular aerobic exercise has positive relation for the reduction of body weight. Because the more you do, the more you benefited. In general, this finding clearly noted that regular aerobic exercise program in high and moderate intensity has a significant effect on the reduction of body weight that resulted following accumulation of execs fat in the body.

Considering the major findings and conclusions of the study, it is important to write the following recommendations. Develop the habits of participating in aerobic training program as part of your life style at least three days per week. Regularly participating in aerobic exercise combined with wellbalanced dietary intake is important. Sport science professionals guide and educate the importance and value of aerobic exercise on the reduction of body weight. Further research on the importance and value of aerobic exercise on the reduction of body weight is highly needed.

\section{ACKNOWLEDGMENTS}

This study would not have been possible without funding support of Jimma University staff research development. The author also thanks the participants and Jimma University fitness center trainers.

\section{REFERENCES}

1. Aromaa A, Koskinen S. Health and functional capacity in Finland. Baseline results of the Health 2000 health examination survey. Publications of the National Public Health Institute, Helsinki, p. 148, 2002.

2. Barness LA, Opitz JM, Gilbert-Barness E. Obesity: genetic, molecular, and environmental aspects. Am J Med Genet A, 2007; 143A(24): 3016-3034.

3. Bjorntop P. International Textbook of Obesity. Report in Sahlgrenska Hospital, Go"teborg, Sweeden, pp. 528, 2001.

4. Boutcher HC. High intensity intermittent exercise and fat loss. J Obes, 2011; Article ID 868305.

5. Burnham JM. Exercise is medicine: health benefits of regular physical activity. Journal of the Louisiana State Medical Society, 1998; 150: 319-323. 
6. Flegal KM, Carroll MD, Johnson CL. Prevalence and trends in obesity among US adults, 1999-2000. JAMA 2002: 288(14): 17231727.

7. Goris AHC, Westerterp KR. Physical activity, fat intake and body fat. Physiology and Behavior, 2008; 94: 164-168.

8. Haslam DW, James WP. Obesity. Lancet, 2005; 366(9492): 1197209.

9. Jeukendrup AE, Wallis GA. Measurement of substrate oxidation during exercise by means of gas exchange measurements. International Journal of Sports Medicine, 2005; 26: S28-S37.

10. Jones DA, Ainsworth BE, Croft JB, Macera CA, Lloyd EE, Yusuf HR.. Moderate leisure time physical activity: who is meeting the public health recommendations? A national cross-sectional study. Archives of Family Medicine, 1998; 7: 285-289.

11. Kraemer WJ, Volek JS, Clark KL. Influence of exercise training on physiological and performance changes with weight loss in men. Medical Science and Sports Exercise, 1999; 31: 1320-1329.

12. Leijssen VA, Saris W, Wagenmakers AJ, Senden JM, Baak MA. Effect of exercise training at different intensities on fat metabolism of obese men. Journal of Applied Physiology, 2002; 92: 1300-1309.

13. Maughan RJ, Rehrer NJ. Gastric emptying during exercise, Sports Science Exchange, 1993; \#46, Volume 6.

14. Osterberg KL, Melby CL. Effect of acute resistance on post exercise oxygen consumption and resting metabolic rate in young women. International journal of sport nutrition and exercise metabolism. 2002; 10(1): 71-81.
15. Sallis JF, Owen N. (1999).Determinants of physical activity, In Physical activity and behavioral medicine. Thousand Oaks, CA: Sage, pp. 110-134, 1999.

16. Stiegler P, Cunliffe A. The role of diet and exercise for the maintenance of fat free mass and resting metabolic rate during weight loss. Sports Medicine. 2006; 36: 239-262.

17. Tall A. 2002. Exercise to reduce cardiovascular risk: how much is enough? England Journal of Medicine, 2002; 347: 1522-25.

18. Tremblay A, Almearas N, Boer J, Kranenbarg EK, Despreas JP. Diet composition and post exercise energy balance. American Journal of Clinical Nutrition, 1999; 112: 121-127.

19. Tremblay A, Simoneau JA, Bouchard C. Impact of exercise intensity on body composition fatness and skeletal musical metabolism. Metabolism, 1994; 43: 814-18.

20. Vuori IM. Health benefits of physical activity with special reference to interaction with diet. Public Health Nutrition, 2001; 4: 517-528.

21. WHO. Obesity: Preventing and Managing the Global Epidemic. Report of a WHO consultation. World Health Organ Tech Rep Ser, World Health Organization, Geneva. pp. i-xii, 1-253, 2000.

22. World Health Organization (WHO). 2003. Obesity and overweight, retrieved 2009-04 27.

23. www.cdc.gov: Target heart rate and estimated maximum heart rate. March 30, 2011. 